\title{
DECAY OF THE RESTORATION RENDER MORTAR OF THE CHURCH OF SAN MANUEL AND SAN BENITO, MADRID, SPAIN: RESULTS FROM OPTICAL AND ELECTRON MICROSCOPY
}

\author{
M.J. Varas ${ }^{\mathrm{a}}$, M. Alvarez de Buergo ${ }^{\mathrm{b}}$, E. Perez-Monserrat ${ }^{\mathrm{b}}$, R. Fort ${ }^{\mathrm{b}}$ \\ a. Departamento de Petrología. Facultad de Ciencias Geológicas. Universidad \\ Complutense de Madrid, C/ José Antonio Nováis 2, 28040 Madrid. SPAIN \\ mjvaras@geo.ucm.es \\ b. Instituto de Geología Económica (CSIC-UCM), Facultad de Ciencias Geológicas, \\ Universidad Complutense de Madrid, C/ José Antonio Nováis 2, 28040 Madrid. \\ SPAIN alvarezm@geo.ucm.es empmon@geo.ucm.es $\underline{\text { rafort@geo.ucm.es }}$
}

\begin{abstract}
The main aim of this research is to determine the reasons for the detachment of a mortar rendering applied to the stone masonry of the Church of San Manuel and San Benito (1903-1910), Madrid, Spain. Two building materials were analysed using optical and electron microscopy: the original limestone dressing affected by intense flaking, and the restoration render mortar used in a relatively recent rehabilitation to 'consolidate' the decayed stone. Results obtained by microscopic techniques and from analysis of the environmental conditions to which the building is exposed made it possible to conclude that 1) the mortar is a good material and compatible with the stone, 2) the render mortar failure was mainly due to its incorrect application and the unsuitable repair technique chosen, and last, 3 ) the materials decay was worsened by the adverse exposure conditions of the upper section of the building (pounding rain and sun radiation).
\end{abstract}

Keywords: restoration render mortar, stone decay, exposure conditions, optical microscopy, SEM 


\section{Introduction}

The Church of San Manuel y San Benito (Madrid, Spain, Fig. 1) was built between 1903 and 1910 by the prestigious architect Fernando Arbós y Tremanti. The Neo-Byzantine structure, an unusual style for Madrid, is characterised by the great variety of natural building materials used, brought from different places around Spain by the country's newly constructed railway network [1]. The building, with an almost Latin-cross floor-plan, is surrounded by eight slender ornamented towers and finished by a copper dome supported by nearly circular drum (16 sides) (Fig. 2). The church has been used for worship, except during the Spanish Civil War (1936-1939), when it saw service as a military headquarters. It currently houses the provincial seat of the Augustinian Order in Spain. The property was declared of Cultural Interest in 1982.

Building materials include: granite, found in the socles of the façades and in the grating that surrounds the building; brickwork walls with a external limestone dressing $13 \mathrm{~cm}$ thick; and marble, found in balusters and columns of the windows (white marble), and in some decorative panels (white, black, red marble). The interior of the church is dressed in Venetian mosaic, and the dome is copper finished.

Since 1975, the building has undergone several restorations. The last (19901995) consisted mainly in consolidating the severely decayed limestone external façades by covering them with a restoration render mortar, followed by the application of a paint and a chemical-protective surface treatment. At the present time, this mortar has severe stability problems and has fallen off in some sections (Fig. 3).

The decay of the limestone is more severe in the upper parts of the building, mainly around the drum, terrace area and small towers. The stone surface is 
weakened due to intense flaking and spalling, which have led to surface stone fragments becoming detached (Fig. 4). This deterioration process has penetrated to $5-6 \mathrm{~cm}$ beneath the stone surface.

Restoration render mortar was used to consolidate the severe decayed stone and to rebuild decorative and architectural components such as mouldings, imposts, cornices, etc., affected by significant volume loss. Between one and six layers of mortar were applied, depending on the amount of stone surface loss in need of rebuilding; its thickness ranges from $4 \mathrm{~mm}$ to $3 \mathrm{~cm}$ (Fig. 5). To the naked eye it appears very porous, with circular-shaped voids. This porosity defines the different layers in the mortar. The render mortar was directly placed on the stone surface, without any steel net or anchorages to support its weight. Both mortar and stone are finished with a yellowish paint and a protective treatment. In many cases, mortar panels or plaques were placed following and coinciding with the limestone dressing joints.

At present, the restoration render mortar is extremely degraded in the drum area, with bulging and fissuring that sometimes lead to the complete detachment of mortar fragments of significant volume (Fig. 3). These breakages are severe in the upper areas oriented W, SW, S and SE (Fig. 2) and are mainly located under the windows level of the drum (Fig. 6) and at the slope change with the roofing of the side chapels (grader area, Fig. 3).

Environmental conditions play an important role as the more deteriorated upper zones coincide with the zones which are more exposed to sun radiation ( $\mathrm{S}$ and SW) and become significantly hotter than environmental temperatures (Fig 2).

The main aim pursued with this research is to determine the causes that have led to the failure of the restoration or consolidation of the external walls of the Church of San Manuel y San Benito, leading to the destabilisation and detachment of 
large fragments of mortar renderings applied to the stone masonry in the previous restoration project. To that aim, the materials, the deterioration processes, the causes of decay (especially the influence of the microclimatic conditions), and the design and execution of the previous rehabilitation have been studied.

In general, the restoration of the church of San Manuel y San Benito involved many different aspects, including recent decay and decay of previous restorations. This study aims to provide data on original and repair materials in order to better specify future repair materials and application methods.

\section{Materials and methods}

Environmental conditions play an important role in the decay processes of this case study. The most decayed areas of the building are the highest ones, around the church drum, which are the most exposed to sun radiation, pounding rain and wind. Those that are worst preserved are oriented towards the SW-S-SE and the S (Fig 2), corresponding to the areas most exposed to sun radiation. To analyse the effect of microclimatic conditions on the materials, temperature and humidity data loggers were installed. Data were collected from both the environment (air temperature and relative humidity) and directly from the materials's surfaces (stone surface temperature; temperature and humidity content in the stone-render mortar contact). These data loggers were placed in three areas with different orientations during winter (from 13 December 2003 to 26 January 2004) and summer season ( from 3 June 2004 to 3 August 2004). The equipment used was:

- Onset Hobo Temperature Data Logger (1996, Computer Corp. S/N 140470), with a standard temperature range of $-20^{\circ} \mathrm{C}$ to $+70^{\circ} \mathrm{C}$ for the logger and of $-40^{\circ} \mathrm{C}$ to $+120^{\circ} \mathrm{C}$ for the sensor; sampling interval ranged from 0.5 seconds to $9.0 \mathrm{~h}$ hours, and an accuracy of $0.2^{\circ} \mathrm{C}-0.4^{\circ} \mathrm{C}$. 
- Onset Hobo Relative Humidity Data Logger (1993, Computer Corp. S/N 111466), with a standard relative humidity range of 25 to $95 \%$, and an accuracy of $\pm 5 \%$.

Both Hobo Data Loggers had a capacity of 1800 measurements readings, and a BoxCar 2.06 software was used for data acquisition and processing.

Wind data (dominant bearing and speed) were provided by station $\# 8$ of the weather and pollution stations of the Surveillance Automatic Network (air quality) of the City Council, Madrid, which is located two hundred metres from the building (from 1 January 2003 to 31 July 2004).

This study mainly sampled two types of materials: the limestone dressing the brickwork structure of the church, and the mortar rendering used to repair the decayed stone in an earlier restoration dated 1990-1995. The paint applied on the stone in the rehabilitation was also sampled, together with a chemical-protective surface treatment visible with microscopic techniques but not to the naked eye. Sampling was carried out by taking flakes (paint), small fragments (stone, mortar) and some mortar-stone cores from earlier adherence tests in the drum area. Artefacts were reduced to the minimum during the sampling process following recommendations suggested by [2] to avoid unnecessary pressure during their extraction (no core drilling was used, either hammer or chisel).

Of a total of 26 samples (stone, render mortar and stone+render), 14 were taken from the SW-oriented façade, 8 from NW, 2 from NE and 2 from SE. Most of the samples were taken from the upper and most deteriorated section of the building (drum and surroundings). For the analysis of the deterioration of both materials, render mortar and stone substratum, samples from altered and unaltered areas were taken in order to compare the results obtained. 
Although a wide range of analytical techniques exist that can give information on this specific type of study, microscopic techniques are very useful and representative [3].

Thin section analysis was done in an Olympus BX 51 petrographic microscope using polarized light technique. Images were acquired using and Olympus DP $12(6 \mathrm{~V} / 2,5 \AA)$ digital camera and proprietary software. Magnifications of the images were $\times 4$ and $\times 10(1 \mathrm{~mm}$ and $500 \mu \mathrm{m}$ scale bar in the micrograph, respectively). For the thin sections preparation [4,5], hand specimens were used. When samples were not sufficiently consolidated (mostly stone and stone-mortar samples), they were vacuum impregnated with an epoxy resin. Sections of these samples were polished (the face to be observed), and the polished face was fixed/glued to a glass slide. The section was then cut again up to $2 \mathrm{~mm}$ thick and further reduced on a thin section grinder to a minimum thickness of 50-100 $\mu \mathrm{m}$ (according to the different interference colours of the existing minerals). The next step was the polishing of the thin section with silicon carbide (for polarising microscope) and alumina (for SEM) of different grain sizes to obtain section thickness as low as $30 \mu \mathrm{m}$.

Alizarin Red S staining was used to differentiate calcite from dolomite [6-8]. Petrographic description of thin sections was performed according to Tucker's descriptive worksheets [9].

The Scanning Electron Microscope (SEM) used was a JEOL JSM 6400, 0.2 to $40 \mathrm{kV}$ acceleration voltage, a beam current of $6 \times 10^{-10} \mathrm{~A}, 10^{-5}$ Torr vacuum conditions, $35 \AA$ A SEM resolution, at a working distance of $8 \mathrm{~mm}$ and $35 \mathrm{kV}$. Images were acquired at $20 \mathrm{kV}$ of acceleration voltage. A coupled $\mathrm{X}$ ray energy dispersive spectrometer (EDS) was used (OXFORD-INCA), with a nominal resolution of 133 
$\mathrm{eV}$ at $5.39 \mathrm{kV}$. Samples were graphite sputtered (15 nm thick cover) using a Balzers Med010 sputter coater (Balzers, Liechtenstein).

Both fragments (around $12 \times 12 \times 12 \mathrm{~mm}$ ) and polished, thin sections were studied by SEM-EDS, in secondary (SE) and backscattered electrons (BSE) mode, respectively. Morphology, structure and texture of the different constituents of the sample were analysed in SE mode (surface rugosity and relief), while BSE mode allowed the determination of the elemental composition of the constituents based on their atomic number.

Cross sections of the samples were analysed: perpendicular to the external surface of both render mortar and stone.

Porosity from both mortar and stone samples was determined through mercury intrusion porosimeter - MIP- (Micromeritics Autopore IV 9500). This automatic instrument measures pore diameters from 0.0055 to $360 \mu \mathrm{m}$, with measurement conditions from atmospheric pressure to 33,000 psia (228 $\mathrm{MPa})$. Samples cores of $10 \mathrm{~mm}$ and at least $30 \mathrm{~mm}$ tall were length.

\section{Results}

\subsection{Environmental measurements}

The environmental temperature in the southern areas of the building can reach a maximum of $49{ }^{\circ} \mathrm{C}$ during summer, and there are daily temperature variations over $33^{\circ} \mathrm{C}$. On these days, the mortar surface can rise to maximum temperatures of $54^{\circ} \mathrm{C}$ and undergo thermal variations over $39^{\circ} \mathrm{C}$. At the mortar-stone contact, the temperatures and the thermal variations are similar to those of the environment, although the humidity is slightly higher ( $31 \%$ in summer) than the environmental humidity (29\%). Although the wind is stable $\left(8 \mathrm{kph}^{-1}\right)$, the dominant 
bearing varies between $220^{\circ}$ (SSW) and $130^{\circ}$ (ESE). The wind directly affects the more deteriorated area of the drum as on rainy days these renderings are soaked by pounding rain. Another factor to be taken into account is the fact that the wind bearing is highly changeable throughout the day, varying between $180^{\circ}(\mathrm{S})$ and $250^{\circ}$ (WSW) during the time of highest environmental temperature (1 p.m. -6 p.m.).

\subsection{Stone substratum}

Under the polarising microscope $(\mathrm{OM})$, the limestone is defined as a biomicrite [10], due to the high percentage of bioclasts. The distribution of such percentage is the following: 60vol\% of bioclasts (foraminifer, echinoderms, bryozoan, mollusca and coralline algae), 10vol\% of siliciclastic particles (quartz), $20-25 \mathrm{vol} \%$ of micritic matrix and $5-10 \mathrm{vol} \%$ of pores (Fig. 7). Some neogenic quartz is observed in pores. The total porosity of the stone obtained by MIP is $16.2 \%$

Macroscopically visible flaking continues at microscopic scale, leading to delamination and increased permeability (Fig. 7a). Fissure width varies from 0.37 $\mathrm{mm}$ at depth, to $0.9 \mathrm{~mm}$ near the surface. This fissuring process affects all the stone constituents (bioclasts, matrix and cement).

The stone is not always covered by the mortar rendering but by a yellowish paint. It is a homogeneous, compact, dark-coloured layer that covers the irregularities of the stone surface and has a thickness ranging from 0.1 to $0.15 \mathrm{~mm}$ (Fig. 7b).

The SEM-EDS study of this calcareous substratum confirms what was observed under the OM. The chemical composition detected is mainly $\mathrm{Ca}$, followed by $\mathrm{Si}$. SEM also made it possible to detect the micro-fissures on the stone surface (Fig. 8). The paint layer, which covers the stone, appears as a poorly consolidated layer with significant porosity in its interior and in contact with the underlying stony 
substratum. Its chemical composition is mainly constituted by $\mathrm{Ti}$ and smaller amounts of $\mathrm{Fe}$ and $\mathrm{Si}$. In addition, there are remains of a thin, superficial film, containing $\mathrm{Si}, \mathrm{Mg}, \mathrm{Al}$ and $\mathrm{Ca}$. At the contact between paint and substratum, porosity is higher.

\section{$\underline{\text { 3.3. Restoration render mortar }}$}

With the $\mathrm{OM}$ it is possible to recognise dolomitic marble aggregates floating within a micro-crystalline mass with a massive aspect and undefined mineralogy. These aggregates are very angular and have a well-defined grain-sized selection consisting of three dominant types: $0.1,0.3$ and $1 \mathrm{~mm}$. The morphology of the air voids is very regular and circular. The voids are located within the micro-crystalline matrix (Fig. 9a). Porosity measured by MIP is $22.2 \%$.

As mentioned earlier, the rendering may have a single layer, although multilayer rendering is more frequent. In the latter case (Fig. 9b), the successive layers which appear are very similar to each other but differ slightly in colouring and thickness, as well as in the amount and morphology of the voids. The more superficial layers are thinner and more compact. The aggregate is in contact and its main axis, together with the axis of the voids, is oriented parallel to the surface of the mortar. The lower, thicker layers are not as compact as the upper layers; therefore, the aggregate is very loose and does not appear in contact with each other. No type of orientation of the main axis can be observed.

The stacking of several layers of mortar is marked by a dark-coloured film, which may be due to a lower porosity than the surrounding material, also linked to the appearance of voids on this film oriented with lengthened morphology (Fig. 9b). The contact between the successive layers is straight but discontinuous due to the 
substantial porosity observed in these areas. This can lead to the selective detachment of the layers of mortar.

A thin film of yellow paint always appears on this mortar, giving the surface a uniform appearance (Fig. 10a). In thin section, the yellow topcoat appears as dark colored layer 150-500 $\mu \mathrm{m}$ thick, covering the irregular mortar surface. Bonding of the topcoat is incomplete, as demonstrated by a bead-chain of air voids on the interface with the substratum. Also, there is a much more substantial porosity which separates the layer of paint on the underlying rendering mortar and is particularly higher in the superficial part of this mortar.

The rendering mortar adheres directly to the stone, which has a high degree of deterioration with superficial cracking (Fig 10b). Voids with lengthened morphology, made up of the coalescence of previous underlying rounded voids, are observed. Fundamentally, these voids appear at the base of the rendering in contact with the stone. Cracking, horizontal and parallel with the surface of the stone, affects the base of the rendering.

The analysis of the single layer and multi-layer mortar with the SEM+EDS, confirms that the aggregate is dolomite (Ca-Mg carbonate) and the binder, whose mineralogy was not detected with the OM, is gypsum, confirmed by both the EDS spectrum indicating the presence of $\mathrm{Ca}$ and $\mathrm{S}$ combined with crystals morphology (dense mass of needle and arrow shaped crystals). This mass contains isolated dolomite crystals which are larger and have more irregular shapes. Pigment and stabilizing agents occur interstitially in the gypsum fabric (Fig. 11). In both cases, compounds containing, on one hand, $\mathrm{Si}, \mathrm{Al}$ and $\mathrm{Mg}$ have been detected in specific areas, while, on the other hand, a uniform distribution of the Ti and smaller amounts of Fe have been seen (Fig. 12). They are detected in the area of contact with the underlying stone substratum (Fig. 8). In the case of the multilayer mortar, they 
appear in the contact zone of the layers of mortar. When there are two layers, these chemical composites (mainly containing $\mathrm{Si}, \mathrm{Al}$ and $\mathrm{Mg}$ ) impregnate the surface of the lower layer and especially affect all the binder (Fig. 12). In this area of contact between layers, the line of lengthened voids previously detected with the OM again appears and affects the base of the layer which is applied last (Fig. 12). These impregnations correspond to the extended dark stains which appear in the OM and which make it possible to differentiate the layers of mortar (Fig. 9).

The layer of paint which covers the surface of the rendering mortar was studied in greater detail through the SEM+EDS. This layer has a variable thickness (50-100 $\mu \mathrm{m})$, a granulated appearance and a Ti dominant chemical composition, containing also $\mathrm{Fe}, \mathrm{Si}, \mathrm{Al}$ and $\mathrm{Ca}$ traces. A thin film (20-30 $\mu \mathrm{m})$ containing $\mathrm{Si}, \mathrm{Al}$ and $\mathrm{Mg}$ covers its surface. The lower interface of the paint layer is quite irregular as it adapts to the irregularities of the surface of the mortar. Substantial porosity was detected in the paint mortar contact area (Fig. 13).

\section{Discussion}

In theory, deteriorated walls can be consolidated by covering them with one or several thin layers of fresh mortar composed of a specific binder, small-sized aggregate and added colouring pigments (mainly containing $\mathrm{Ti}$ and $\mathrm{Fe}$ ). Primer coat impregnations were applied on the mortar layers interfaces to improve mortar properties (adherence, rigidity, etc). The wall is previously reinforced with a rehabilitating steel net in order to provide support for the weight of this rendering [11]. This type of intervention leads to a relative improvement in the strength and stiffness of the wall. These actions can be negative if the mortar is not well-designed and, especially, if the execution of the repair technique is incorrect [12,13]. The 
mechanical performance of a wall consolidated with this technique will depend on the distribution and the amount of rendering mortar used and on the number and thickness of the layers of mortar applied. Both these parameters determine the maximum load possible (maximum tolerable weight). However, the effect of this reinforcing technique relies on the bond strength at the interface of the wall substratum with the mortar render $[12,13]$.

One important question not addressed when the intervention technique was designed was why deterioration was located in the upper areas of the W, SW, S and SE of the building and affected all the natural construction material in these zones. As an in-depth study was not carried out on these forms of deterioration, the problems reoccurred in the same zones and affected the new materials (rendering mortars) applied during the 1990-1995 rehabilitation.

In short, these deterioration processes are influenced by a number of environmental and material factors, which together affect all the construction materials. As the materials are quite porous, continuous exposure magnifies the deleterious effects of weather in this area. The construction materials located in the W, SW, S and SE undergo severe deterioration as they coincide with the zones of highest temperatures, strong thermal variations, prevailing winds and pounding rain. Under these conditions, the original stone and the artificial stone used in the restoration deteriorate more quickly.

Furthermore, the types of deterioration which affected the original stone were not taken into account. Therefore, the new rendering mortar was applied to an original stone which had not been restored (i.e. removing the decayed surface or consolidating it). This led to a very weak area between the layer of mortar and the original healthy stone, as confirmed by microscopic techniques. The weakened zone 
of natural stone is intensely cracked, more porous and often comes down when the mortar covering becomes detached.

Consequently, due to the prevailing environmental conditions in the zone and the poor state of the supporting stone, this mortar did not respond properly and underwent cracking, bulging, collapse and detachment. The strong rigidity and resistance provided by this rendering were overcome by the continuous episodes of hydric and thermal expansion experienced since 1995. The combined action of dampness in the mortar and high temperatures caused the mortar to undergo thermal and hydric expansion, which, although not intense, were sufficient to generate stresses which led to processes of deformation and displacement of the mortar rendering.

In addition, although the rendering mortar used (dolomite marble aggregate and gypsum binder) is material of acceptable quality as regards petrographic, mineralogical and petrophysical characteristics, the execution was not properly carried out:

a) This mortar was applied in one or several layers with a thickness which varied from $4 \mathrm{~mm}$ to $3 \mathrm{~cm}$. The $3 \mathrm{~cm}$ thickness brings substantial weight to bear on the mortar, adhering directly to the original altered stone with no kind of mesh or steel or fibreglass anchoring to enable secure fixing. In areas of maximum thickness, the surface of the altered stone does not support the weight of the mortar, and the rendering mortar together with the most superficial part of the supporting stone become detached. This detachment of the part of the stone stuck to the rendering mortar is attributable to the high degree of adherence and rigidity of the mortar-stone interface, probably due to the application of primer coats in such interface (detected in the dark layers containing $\mathrm{Si}, \mathrm{Al}$ and $\mathrm{Mg}$ by means of EDS analysis). That is why 
the detachment, mainly due to continuous episodes of hydric and thermal expansion, does not occur on the interface between the two materials, but rather in the original stone substratum weakened by alteration.

b) In many cases, the design of the rendering follows the division of the stone support, thus, the coincidence of the joints with the areas where the mortar is thinner (joints of the facing panels) implies the presence of stress. This stress leads to the appearance of horizontal and vertical cracks through which rainwater infiltrates. The internal dampness of the mortar, together with high temperatures, cause expansion which leads to the bulging of the rendering in the central area, the complete detachment of the stone support, superficial cracking of the most deformed area of the rendering and, finally, the detachment of rendering plaques which leaves the stone uncovered.

c) Microscopic techniques show the presence of primer coats applied in the stone-mortar and mortar-mortar interfaces and the existence of substantial porosity (entrapped-air) have prejudiced the proper conservation of the material. This was the working method used for the mortar rendering of the stone wall in the rehabilitation of this building.

Two weak factors have been detected at the base of this mortar and these could lead to its detachment. First, the existence of the lengthened voids at the base of the rendering in contact with the stone, due to the large number of air bubbles which were trapped during the application of the mortar on the stone (entrapped air). The second factor is cracking, horizontal and parallel with the surface of the stone, which affects the base of the rendering. The cracks are related to the peeling which appears on all the ashlars. Under these conditions, the rendering mortar on this stone substratum becomes structurally and texturally unstable. This leads to serious weakening of the stone surface and the mortar base, which can entail materials loss 
due to the detachment of the rendering mortar and the superficial part of the stone. These observations verified that no measures had been taken to restore the stone before the rendering mortar was applied. Regarding the paint on the mortar, its external section is also affected by flaking, with a weakened area in contact with the stone due to the dissolution processes of the stone constituents. This section leads to stone breakage and the paint layer breaking off (Fig. 7b, 10a).

With respect of environmental conditions affecting the materials decay, rainwater penetrating through cracks in some parts of the rendering dampens the interior of the mortar. The spread of this dampness throughout the interior of the mortar is the result of a huge porosity network, located between the contact areas of the layers of mortar and between the mortar and the surface of the supporting stone. This porosity is due to the entrapped air inside the layers of fresh mortar during its application.

Furthermore, this dampness is retained inside the mortar by a number of impregnable barriers (primer coats). These barriers impede the evaporation of the humidity from the deepest layers. Thus, the humidity retained increases in relation to the thickness of the rendering and increases its structural destabilisation.

Consequently, the excess weight (the humidity retained in the interior of the mortar plus the substantial thickness of the mortar) which must be borne by a very deteriorated stone surface, together with hostile environmental conditions (principally the high temperatures which can be reached on the surface of the mortar itself, with temperatures above those of the environment.), conditioned the hydric and thermal expansion processes which affected the exterior rendering from 199095. These deterioration processes have led to bulging, cracking, collapse and detachment of the rendering mortar since 1995. 


\section{Conclusions}

- The most decayed area of the building is the Southern façade (from W to SE) of the circular drum. The original stone, as well as the mortar render used in the rehabilitation project, deteriorate here more quickly due to the fact that this zone undergoes the highest temperatures, strong thermal variations, prevailing winds and pounding rain. The existing fissures in the mortar render favour rainwater infiltration and expansion processes, with following bulging, cracking and plaques detachment.

- The masonry limestone is a biomicrite (porosity of $16.2 \%$ ). Its macroscopically visible flaking continues at microscopic scale. The stone was rendered to consolidate it and to stop its severe decay. When it is not covered by this mortar render, it is finished with a yellowish paint.

- The mortar render adheres directly to the decayed stone with no anchorage system; it is a gypsum mortar with marble aggregates (dolomite) colouring pigments (containing $\mathrm{Fe}$ ), and a porosity of $22.2 \%$ due to the great amount of air voids. The render is usually a multi-layer mortar with a thickness ranging from $4 \mathrm{~mm}$ to $3 \mathrm{~cm}$; along the mortar layers interfaces there is an alignment of oriented air voids that creates weakness planes (possible detachment planes), as well as dark areas that correspond to primer coat applications, which also favour detachment through the underneath decayed stone. A thin film of yellow paint always appears on top of the mortar render (Ti and Fe composition), with a high number of air voids in the paintmortar and paint-stone interfaces. A thin film attributable to a finishing protective impregnation (water-repellent) covers the paint layer.

From the study carried out mainly using microscopic techniques, it can be concluded that the failure of the rehabilitation of the Church of San Manuel y San 
Benito was due not only to the flawed design of the operation but also to the faulty execution of the repair technique chosen (fresh rendering mortar).

To sum up, although the mortar is of good quality and is compatible with the stone support, it has performed poorly due to the inadequate treatment and preparation of the stone support (not removing the decayed surface or consolidating it before the mortar application), and adverse environmental conditions. The damage directly affects the modern mortar (also the decayed stone surface coming down with the detached of the covering mortar), and the general state of the building, and deterioration has generally worsened since the rehabilitation in 1995, with bulging, cracking, collapse and detachment of the rendering mortar since then.

\section{Acknowledgements}

This study has been financed by a research contract with the Parish-Church of San Manuel y San Benito, Madrid (Augustinian Order), and by a "Ramon y Cajal" contract (MAdB) (Ministry of Education and Science).

Thanks are given to the "Luis Bru" Electron Microscopy Research Support Centre of the Universidad Complutense de Madrid (UCM), and also to the Linguistic Office of the Centre of Modern Languages of the Complutense University of Madrid, for the proofreading and revising of the paper. 


\section{References}

[1] Gomez-Heras M, Fort R. Location of quarries of non-traditional stony materials in the architecture of Madrid: The crypt of Santa María la Real de la Almudena. Materiales de Construccion 2004;54:31-47.

[2] Crumbie A.K. SEM Microstructural studies of cementitious materials: sample preparation of polished sections and microstructural observations with backscattered images. Artefacts and practical considerations. Proc $23^{\text {rd }}$ Int Conf on Cement Microscopy, Albuquerque, NM, April 29 - May 3; 2001.

[3] Hughes JJ, Cuthbert SJ. The petrography and microstructure of medieval lime mortars from the west of Scotland: Implications for the formulation of repair and replacement mortars. Materials and Structures 2000;33:594-600.

[4] Humphries DW. The preparation of thin sections of rocks, minerals and ceramics. Oxford, UK: Royal Microscopical Society, Oxford Science Publications, Microscopy Handbooks 24; 1992, 83 p.

[5] Ques J, Tritlla J. Introducción a las técnicas de preparación de láminas delgadas. In: Melgarejo JC, editor. Atlas de Asociaciones Minerales en Lámina Delgada. Publicaciones de la Universidad de Barcelona \& Fundación Folch. Barcelona; 1997, p. 33-7.

[6] Friedman GM. Identification of carbonate minerals by staining methods. Journal of Sedimentary Petrology 1959;29:87-97.

[7] Evamy B.D. The application of a chemical staining technique to the study of dedolomitisation. Sedimentology 1963;2:164-170.

[8] Miller J, editor. Microscopical techniques: I. Slices, slides, stains and peels. In: Tucker ME, editor. Techniques in Sedimentology. Oxford: Blackwell Scientific Publications; 1988, p. 86-107. 
[9] Tucker ME, editor Sedimentary Petrology: an introduction to the origin of sedimentary rocks. $3^{\text {rd }}$ ed. Oxford: Blackwell Scientific Publications; 2001: $11-20,110-114$

[10] Folk R.L. Spectral subdivision of limestone types. In: Ham WE, editor. Classification of Carbonate Rocks. American Association of Petroleum Geologists; 1962, 62-85.

[11] Valluzi RM, Binda L, Modena C. Experimental and analytical studies for the choice of repair techniques applied to historic buildings. Materials and Structures 2002;35:285-292.

[12] Ashurst J, Ashurst N. Mortars, Plasters \& Renders. Practical Building Conservation Vol 3. English Heritage Technical Handbook. Aldershot, Hampshire, United Kingdom: Gower Technical Press; 1989.

[13] Binda L, Gambarotta L, Lagomarsino S, Modena C, Segnalini O. Montesanto e Roccanolfi centri storici a rischio (available only in Italian). Bollettino Italia Nostra 1999;355:5-8. 


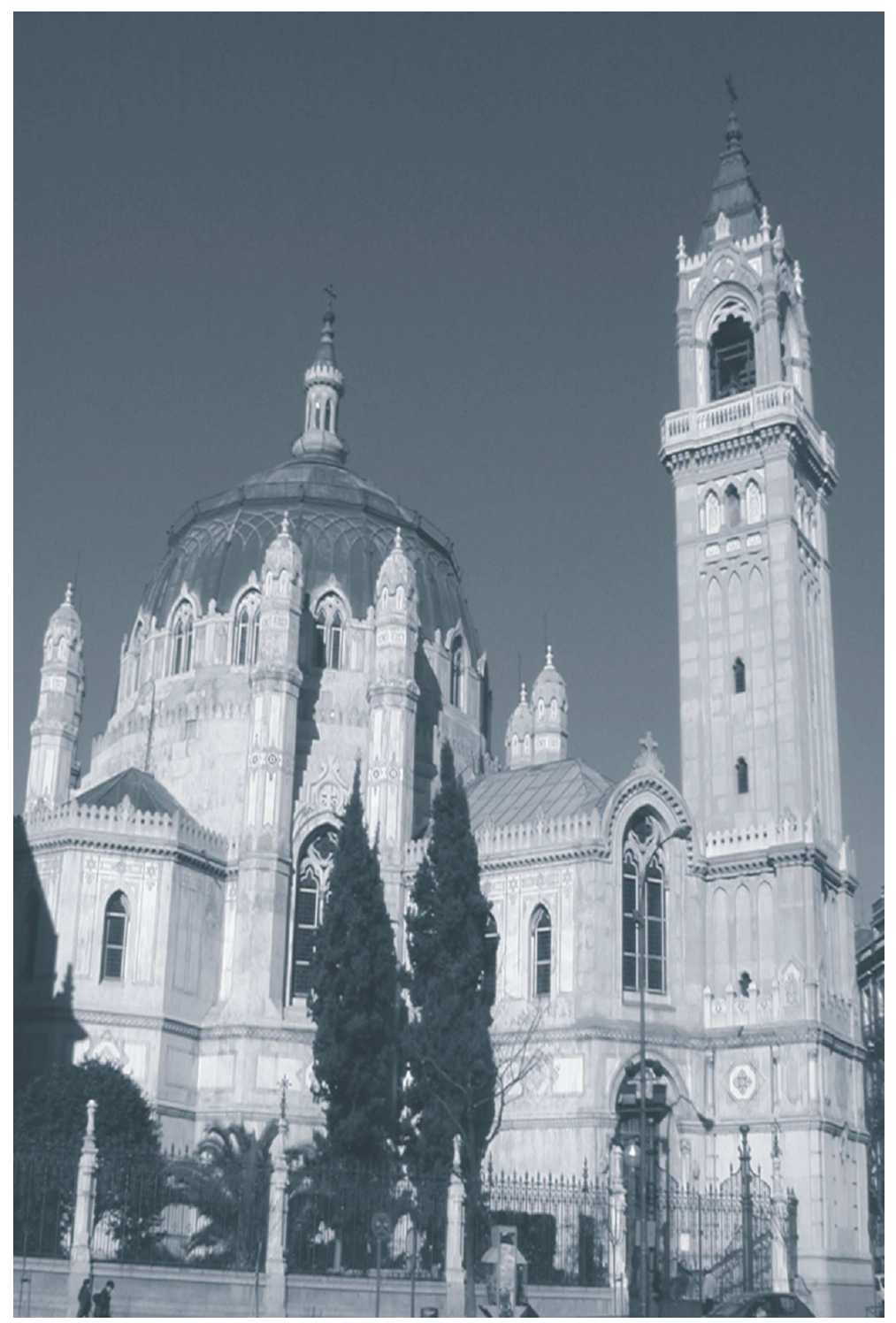

Fig. 1. General view of the building. South façade. 


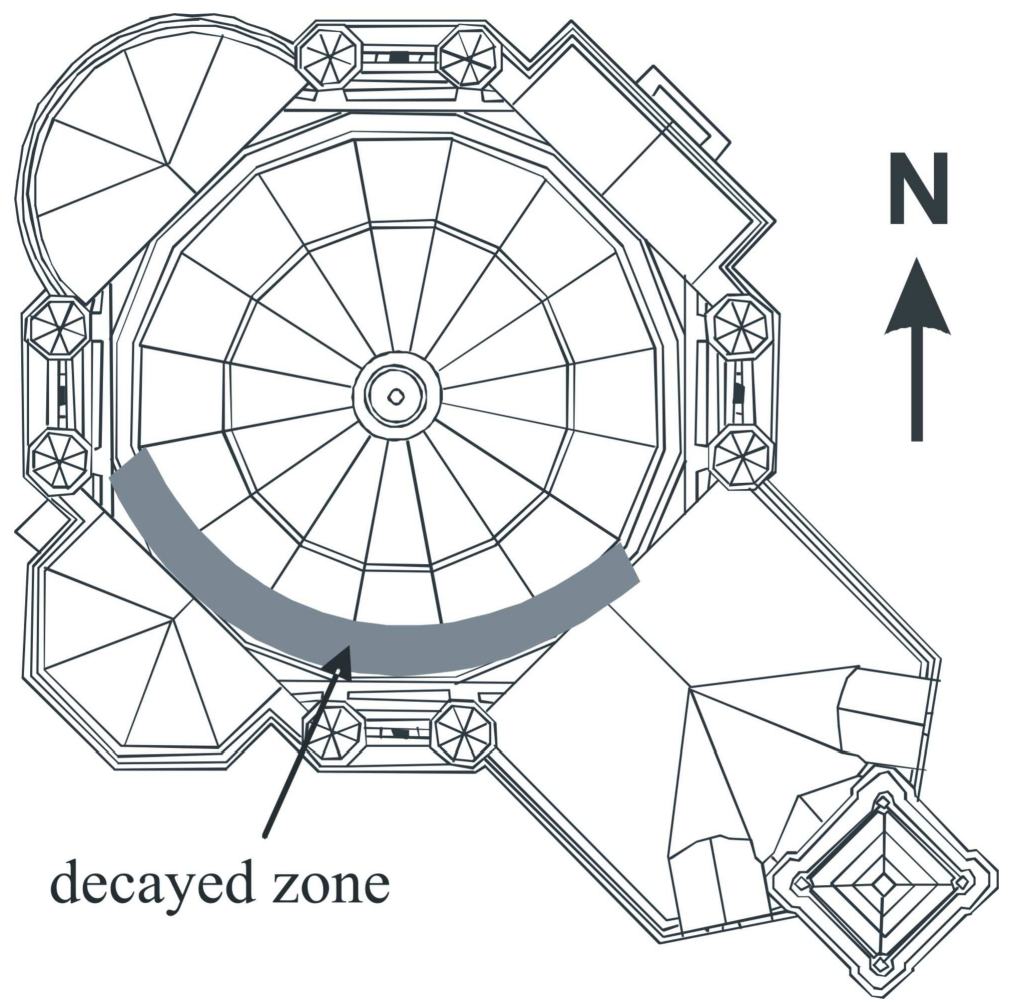

Fig. 2. Ground plan of the building.

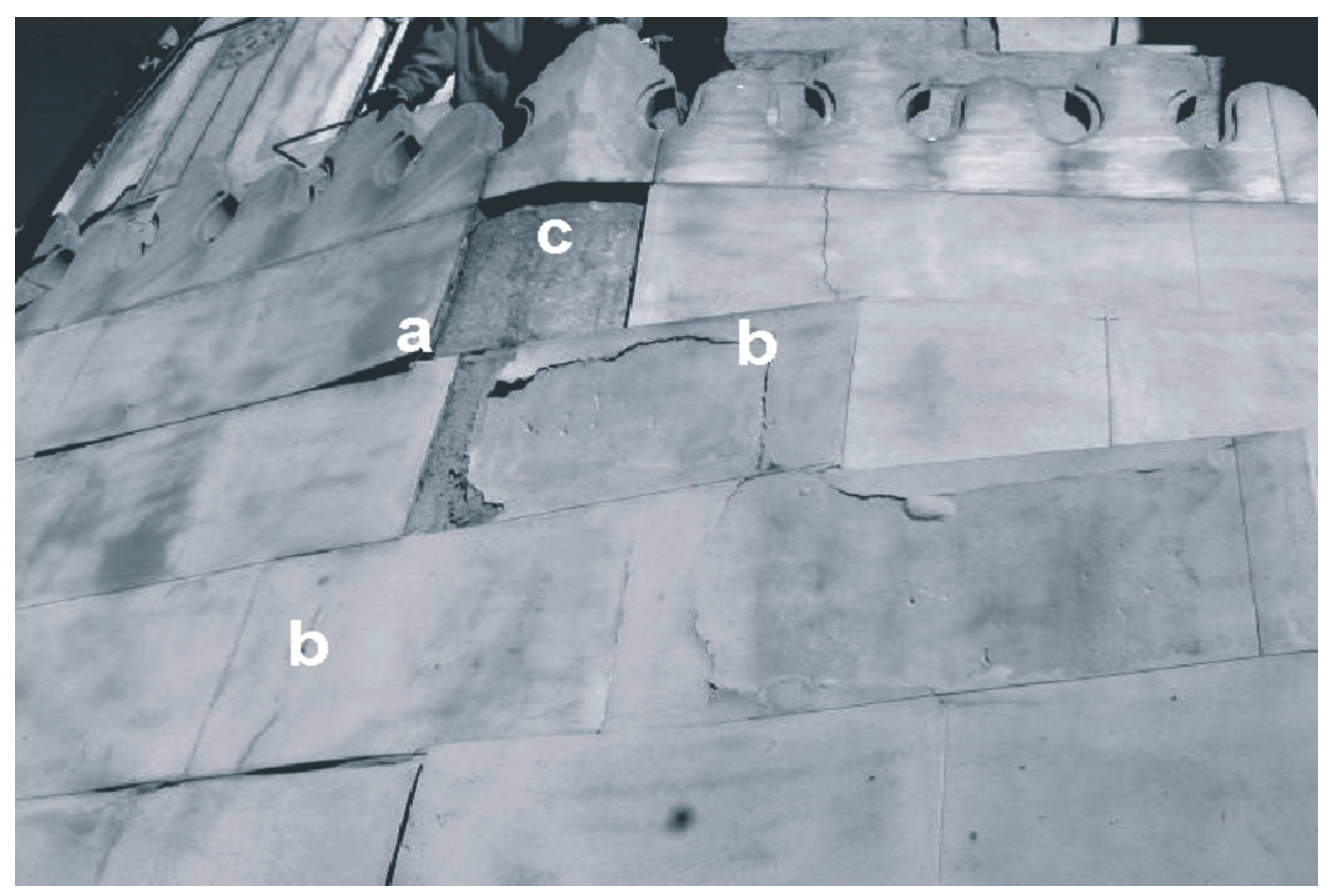

Fig. 3. The fissure pattern (a) in the rendering mortar follows the original stonework joints. The increasing bulging that results on mortar cracks (b) and, later on, on themortar detachment (c). 


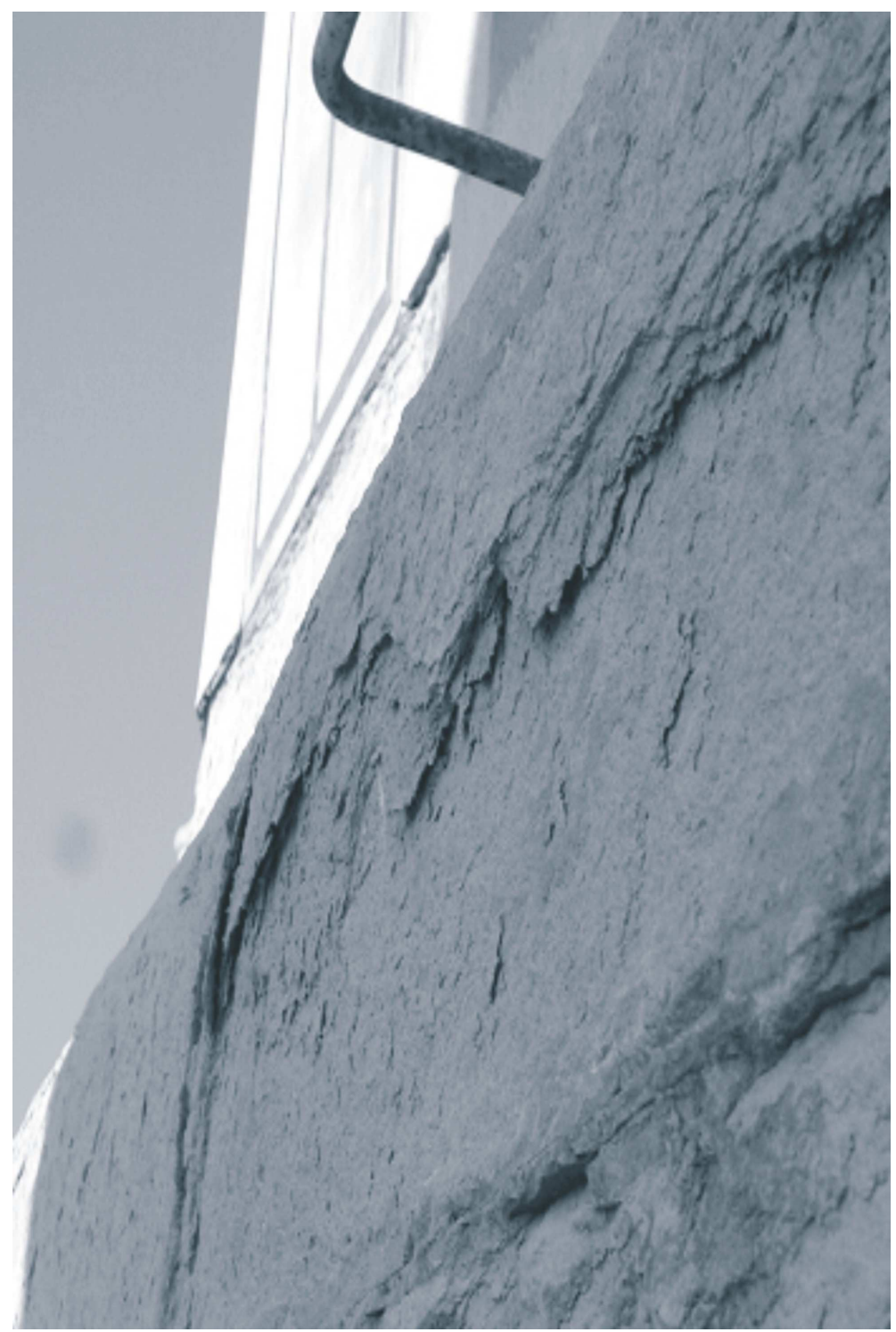

Fig. 4. The stone under the rendering mortar is severestronglyly decayed. The most frequent deterioration forms are flaking and spalling, involving a great amount of surface volume loss. 

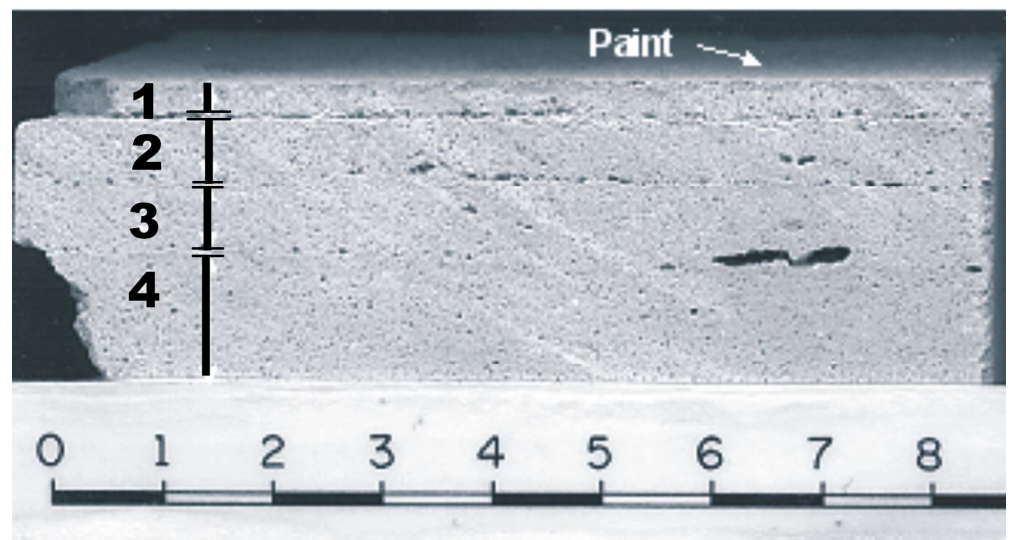

Fig. 5. Multilayered mortar applied on the surface of the decayed stone. The interface among the different layers is characterized by the presence of a high number of air voids.

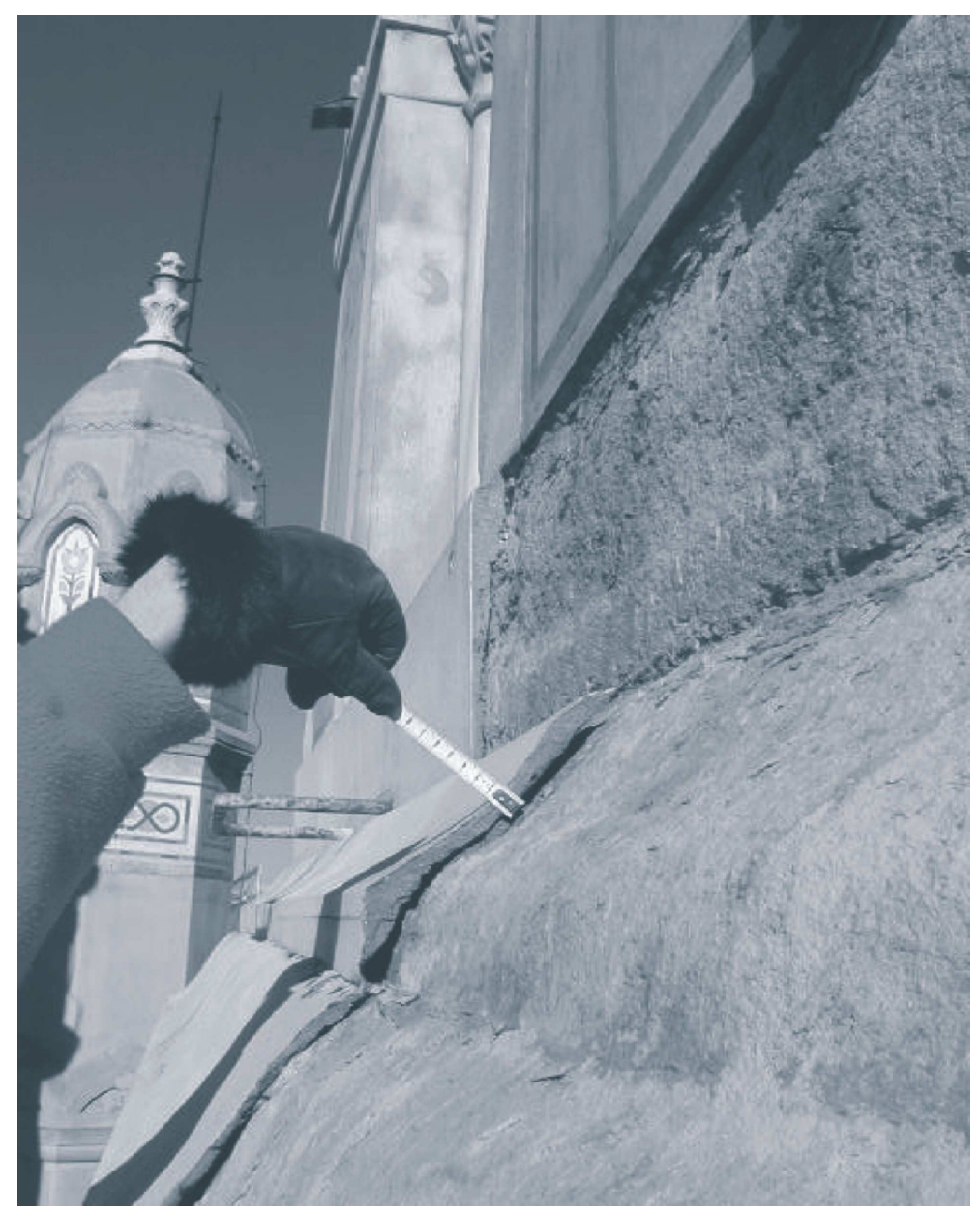

Fig. 6. The mortar applied on the surface of the decayed stone has been used to reproduce many architectural and ornamental elements that were lost. 

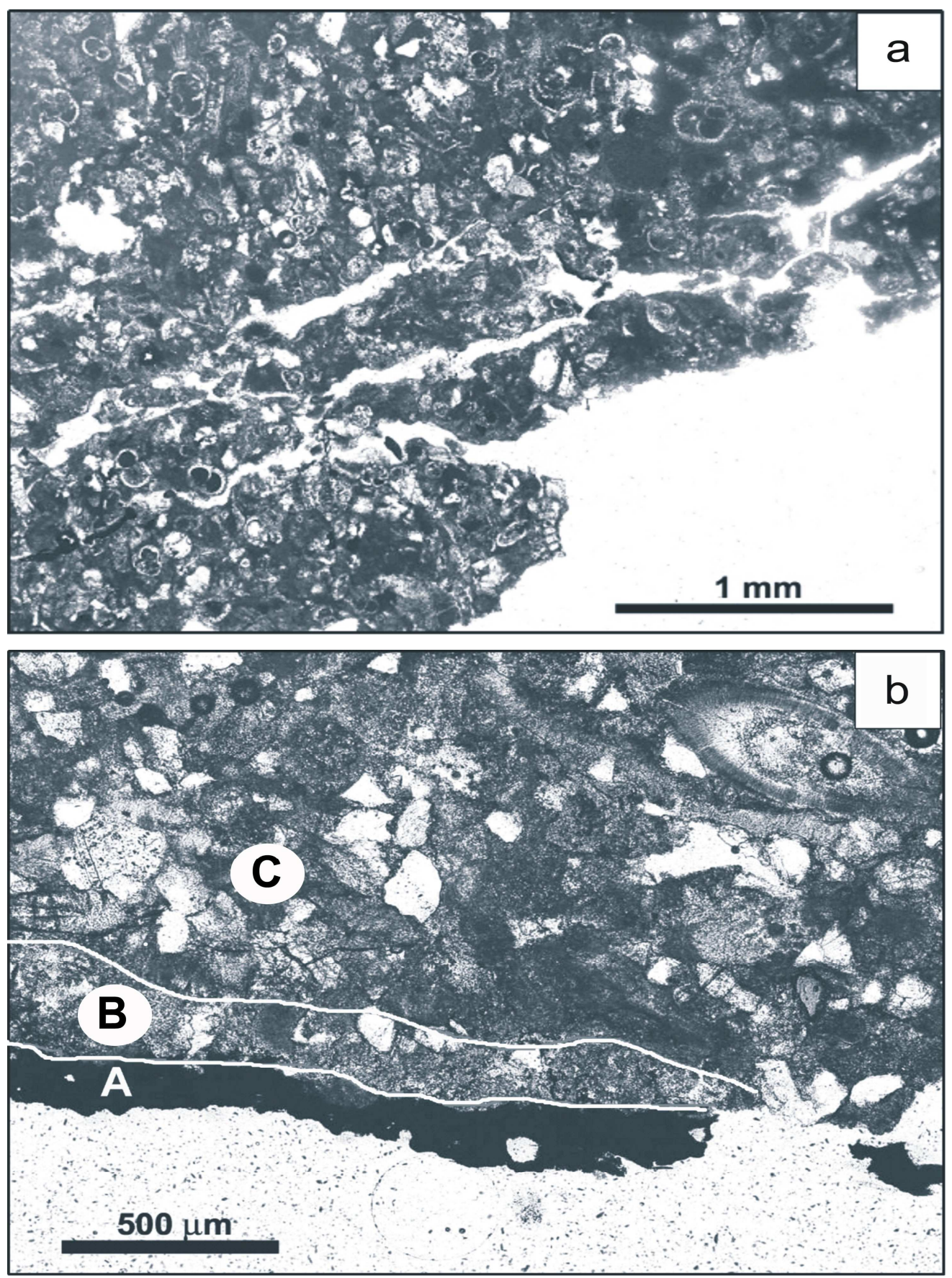

Fig. 7. Micrographs under OM (plane polarized light, PPL) of the stone. a) The presence of fissures parallel to the external surface is the responsible for the flaking of the stone surface. c) Weakness area (B; high porosity) under the paint layer (A) that may occur directly on the stone $(\mathrm{C})$. 

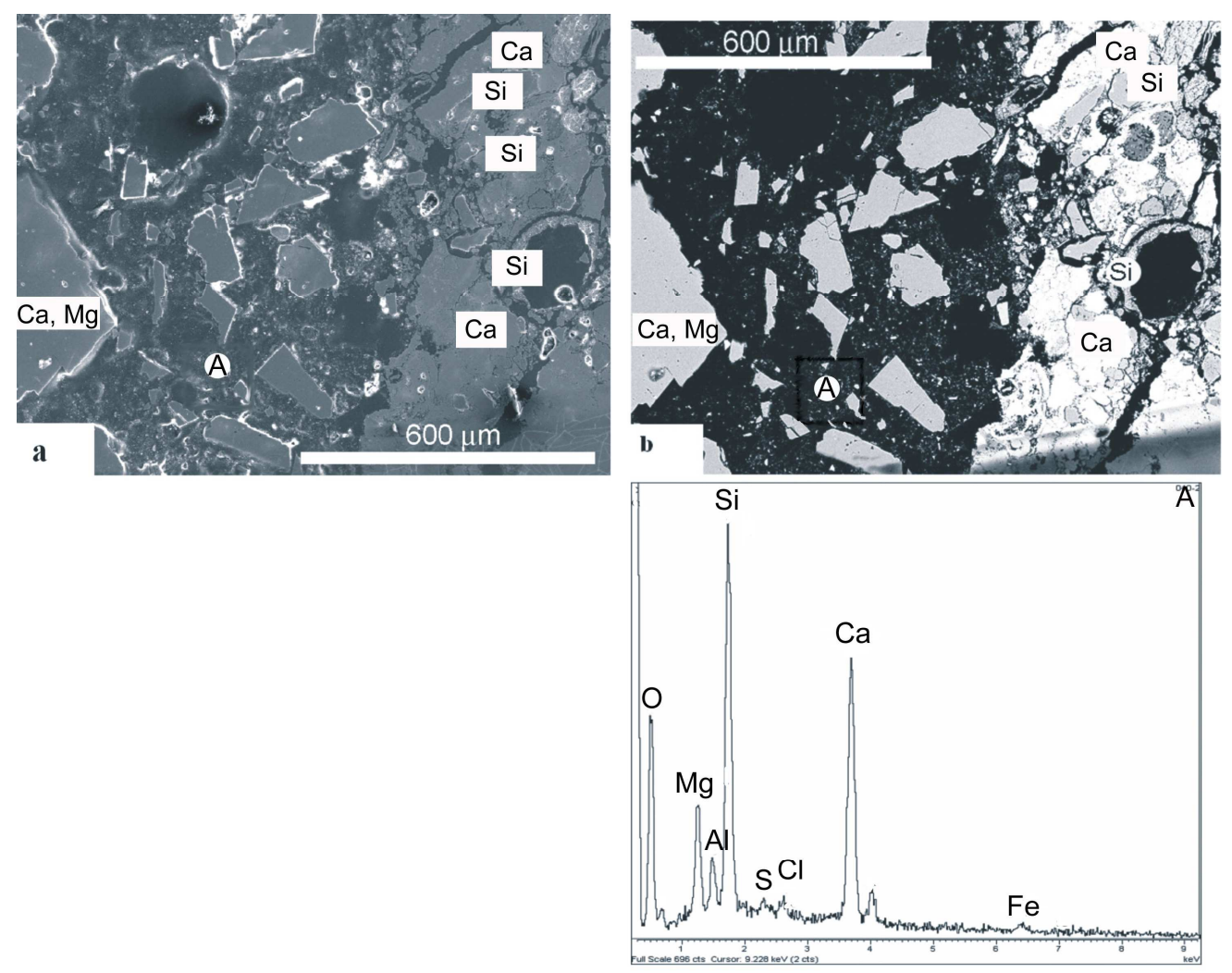

Fig. 8. SEM micrographs (secondary electrons mode) (a) and backscattered mode (b) of a polished section of the contact between the highly fissured limestone (right) and the rendering mortar (left). The stone contains $\mathrm{Ca}$ and $\mathrm{Si}$. The mortar is constituted by a dolomitic aggregate $(\mathrm{Ca}$ and $\mathrm{Mg}$ ) and a binder (A -point analysis) composed of gypsum $(\mathrm{Ca}$ and $\mathrm{S})$, prime coat $(\mathrm{Si}, \mathrm{Al}$ and $\mathrm{Mg})$ and colouring pigments $(\mathrm{Fe})$. 

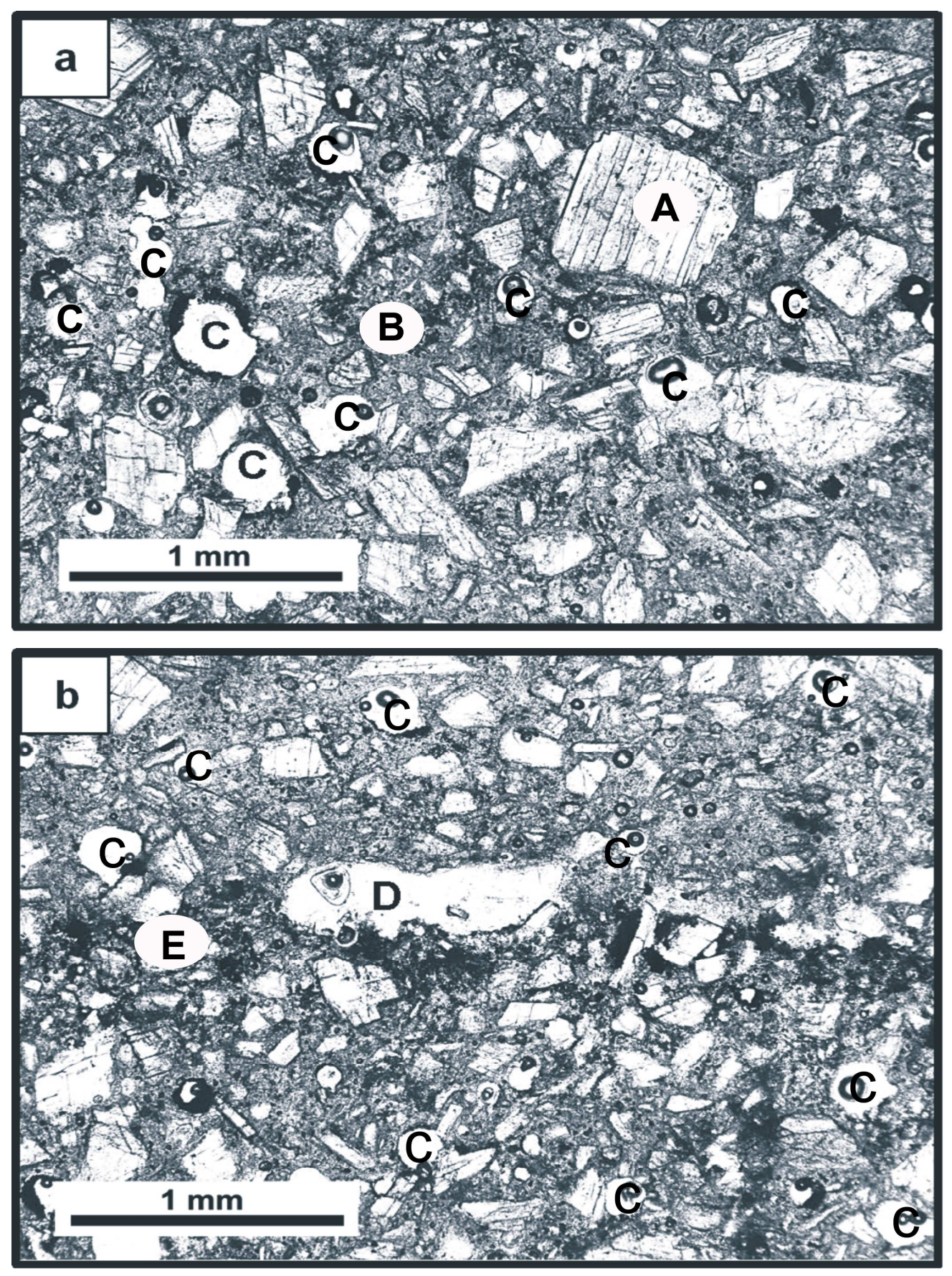

Fig. 9. Micrographs under OM (plane polarized light, PPL) of the rendering mortar. a) General appearance of the mortar (A-dolomitic aggregate, B-binder, C-air voids). b) The different layers of the mortar are limited by the presence of elongated voids (D) and by a dark layer (E). 

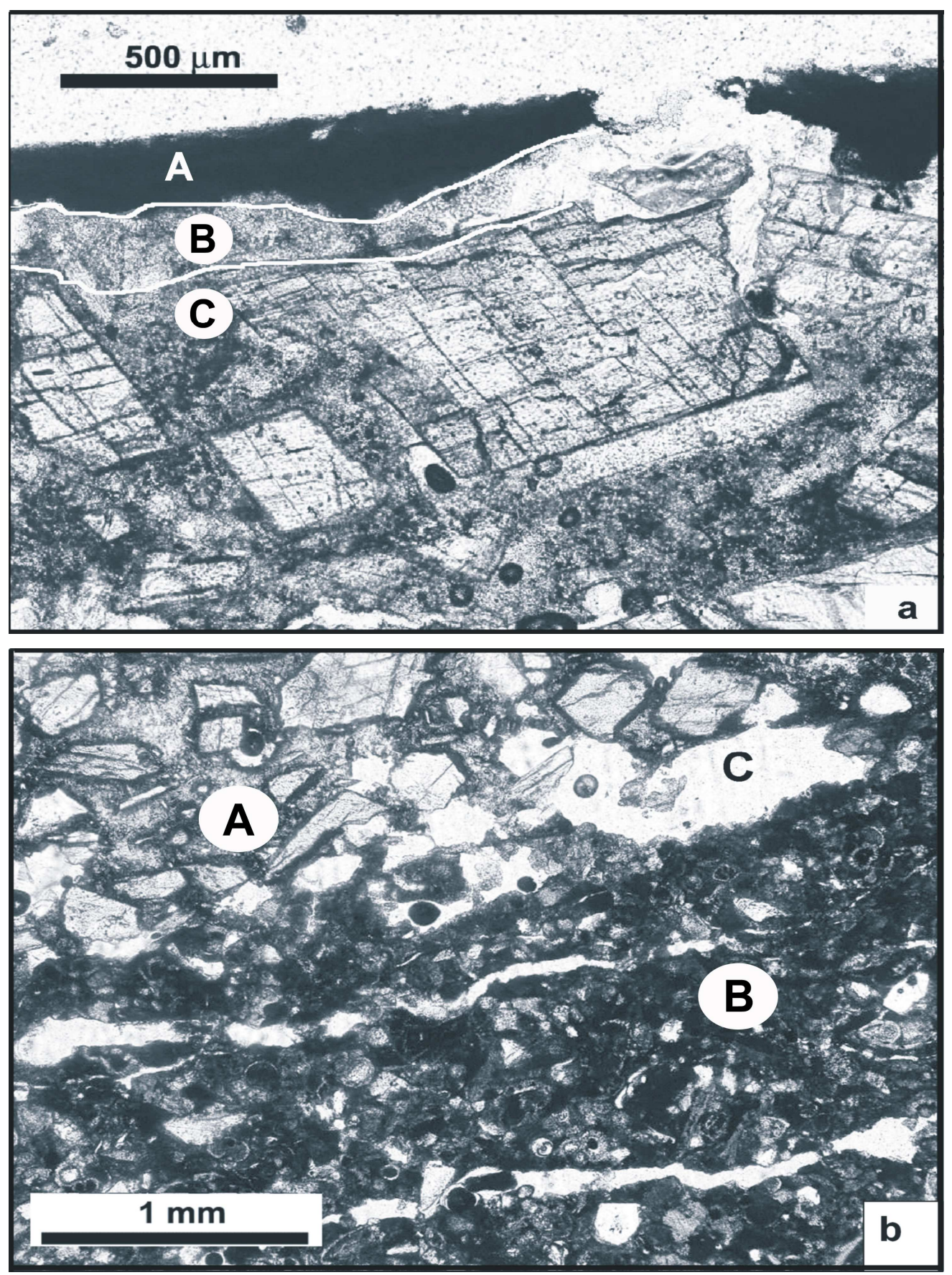

Fig. 10. Micrographs under OM (plane polarized light, PPL) of: a) The paint layer (A) that is also on the modern rendering mortar (C). There is a weakness area in the interface between the paint layer and the most external section of the mortar (B; high porosity). b) The contact area between the strongly fissured natural stone (B) and the rendering mortar $(\mathrm{A})$. There are elongated voids along this contact plane $(\mathrm{C})$. 

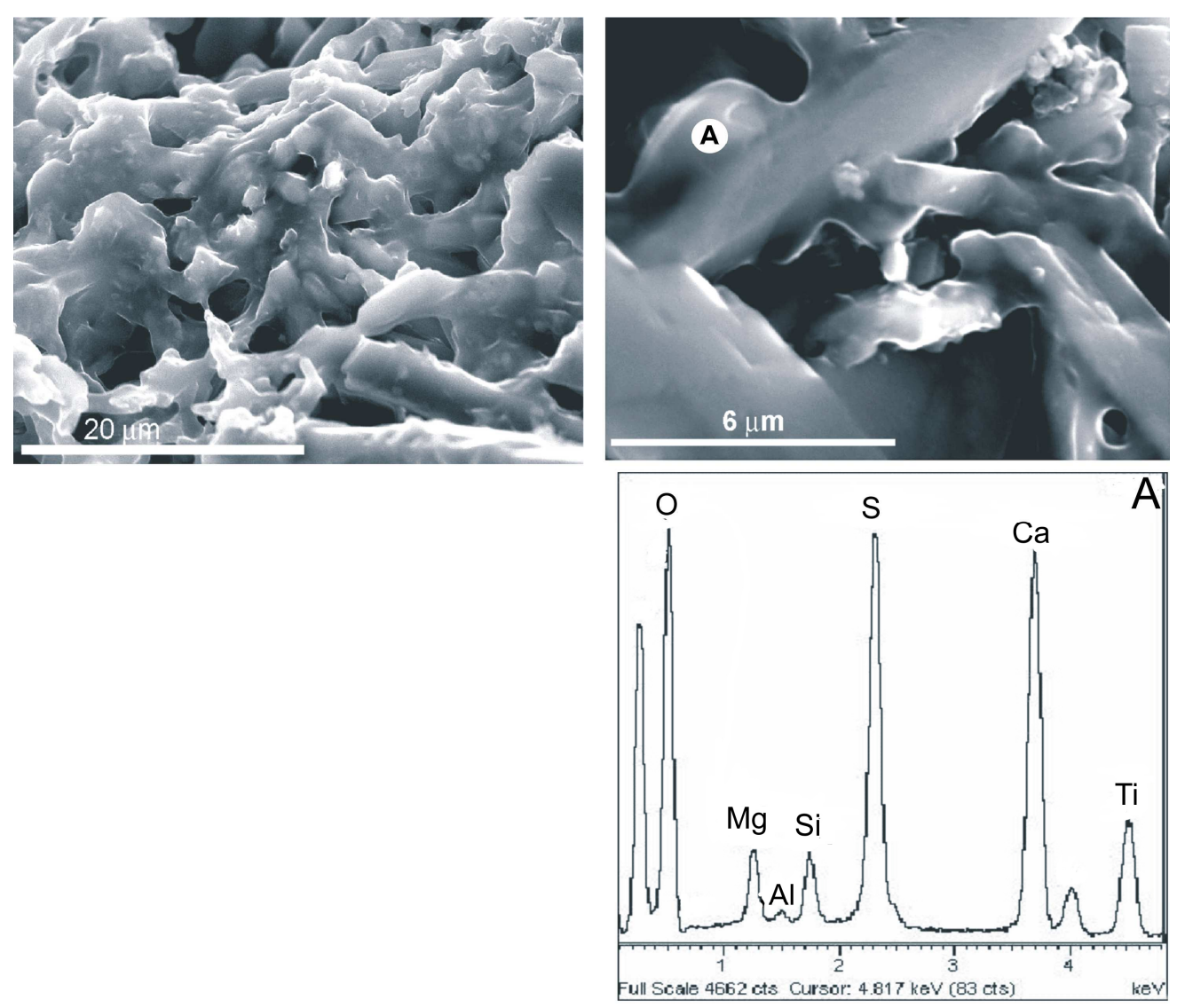

Fig. 11. SEM micrographs (secondary electrons mode) and EDS spectrum of a fragment of the rendering mortar. The gypsum crystals present growing enlargement due to the impregnation with prime coats $(\mathrm{Si}, \mathrm{Al}$ and $\mathrm{Mg}$ ) and to the pigments ( $\mathrm{Ti}$ and $\mathrm{Fe}$ ) added to the binder. Point analysis (A). 


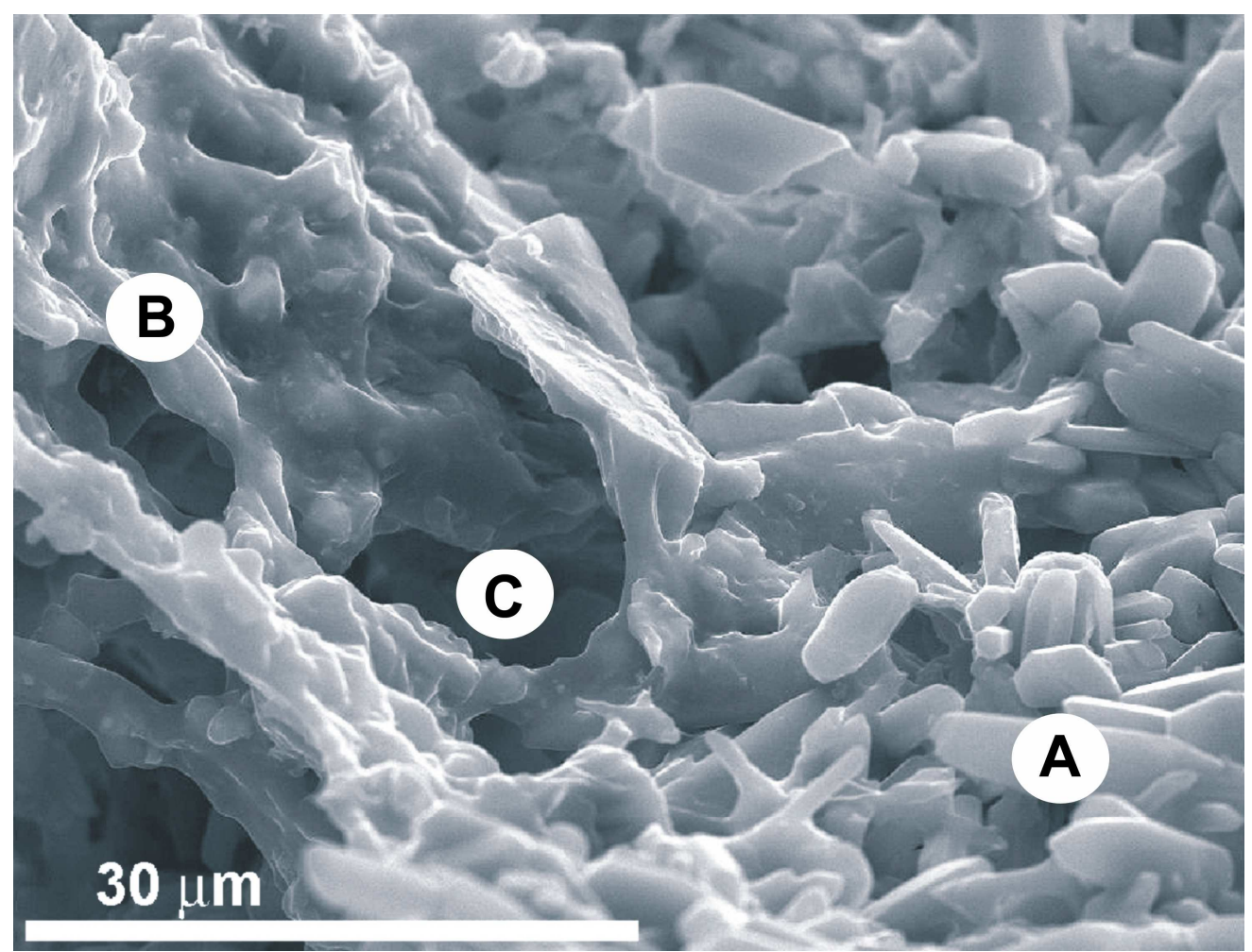

Fig. 12. SEM micrographs (secondary electrons mode) of a fragment of the rendering mortar. A-Gypsum crystals without prime impregnation (mortar, upper layer), B-Gypsum crystals with prime impregnation (mortar, lower layer) and C-Air voids in between both layers. 


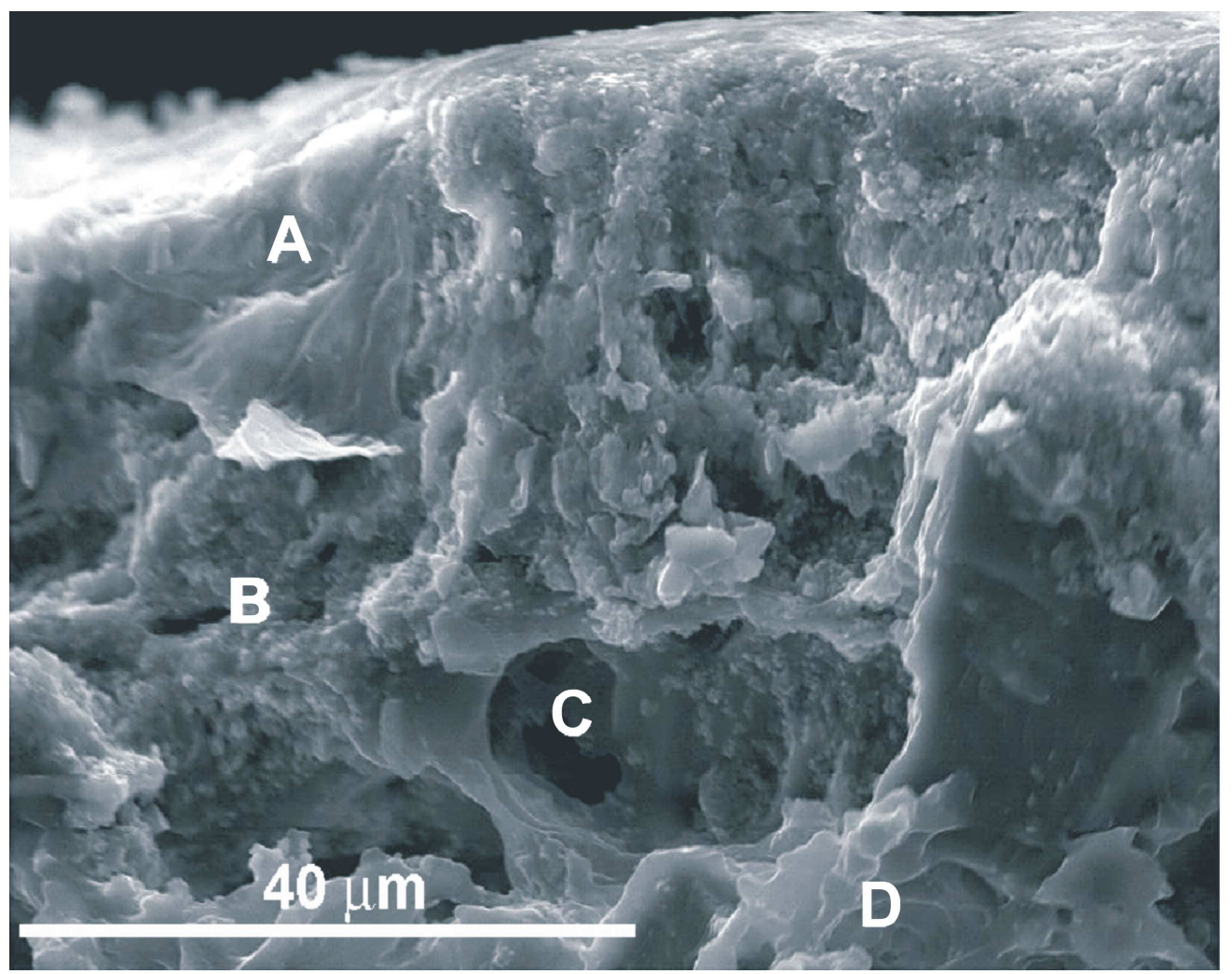

Fig. 13. SEM micrographs (secondary electrons mode) of a fragment of paint, surface layer. A-Film corresponding to a water-repellent treatment, B-Paint layer, CVoids and D-Rendering mortar. 